\title{
THE LATTICE OF $l$-GROUP VARIETIES
}

\author{
BY
}

\author{
J. E. SMITH $^{1}$
}

\begin{abstract}
For any type of abstract algebra, a variety is an equationally defined class of such algebras. Recently, attempts have been made to study varieties of lattice-ordered groups ( $l$-groups). Martinez has shown that the set $\mathbf{L}$ of all $l$-group varieties forms a lattice under set inclusion with a compatible associative multiplication. Certain varieties $\delta_{p}$ ( $p$ prime) have been proved by Scrimger to be minimal nonabelian varieties in $L$. In the present paper, it is shown that these varieties can be used to produce varieties minimal with respect to properly containing various other varieties in $\mathbf{L}$. Also discussed are the relations among the $\delta_{n}(n \in N)$, and it is established that all infinite collections of the $\delta_{n}$ have the same least upper bound in $\mathbf{L}$. Martinez has also classified l-groups using torsion classes, a generalization of the idea of varieties. It is proved here that $\mathbf{L}$ is not a sublattice of $\mathbf{T}$, the lattice of torsion classes.
\end{abstract}

1. Introduction. As the study of varieties of groups has proved to be such a useful tool in group theory, it is natural that varieties of lattice-ordered groups (l-groups) are of interest to those concerned with l-group theory. The first works to appear in this area dealt mainly with specific varieties. Weinberg's work on abelian l-groups [14] led to the conclusion that the abelian variety $\mathscr{Q}$ is the smallest nontrivial l-group variety. Martinez [9] considered a family of varieties $\mathfrak{L}_{n}(n \in N)$ defined by the equation $x^{n} y^{n}=y^{n} x^{n}$. Scrimger [13], working within the $\mathfrak{L}_{n}$ showed that certain varieties $\delta_{p} \subseteq \mathfrak{L}_{p}$ cover $\mathcal{Q}$ for all primes $p$. Holland [6] proved that the variety $\Re$ of normal-valued $l$-groups contains every proper $l$-group variety.

A more comprehensive study of $l$-group varieties was made by Martinez [10], in which the set $\mathbf{L}$ of all $l$-group varieties was shown to form a complete lattice, using containment as the partial order, with a compatible multiplication. The present paper is an attempt to extend the work on the structure of $\mathbf{L}$ and to further investigate the role of the $\delta_{n}$ 's within this lattice. The notation to be used will follow the lines of that used by $H$. Neumann [12].

Starting near the top of the lattice $\mathbf{L}$, we show in (2.2) that $\mathscr{N}$ is finitely join-irreducible. At the other extreme of the lattice, it is first shown in (3.11) and (3.12) that the sublattice of $\mathbf{L}$ generated by $\left\{\delta_{p} \mid p\right.$ is prime $\}$ is lattice-isomorphic to the lattice of finite sets of primes. However, this isomorphism does not extend to the complete sublattice generated by $\left\{\delta_{p} \mid p\right.$ is prime $\}$, as it is shown in (3.14) and (3.17) that the join of any infinite collection of $\delta_{n}$ 's is the variety generated by $Z$ Wr $Z$.

Received by the editors August 24, 1977.

AMS (MOS) subject classifications (1970). Primary 06A55, 08A15; Secondary 06A23, 06A35, 06A50.

Key words and phrases. Lattice-ordered group, variety, lattice, Scrimger variety, torsion class.

${ }^{1}$ This paper is a portion of the author's doctoral dissertation, written under the direction of $\mathrm{W}$. Charles Holland. 
The other main result of this paper deals with the relationship between $\mathbf{L}$ and $\mathbf{T}$, the set of all torsion classes of $l$-groups. It has been shown by Martinez [11] that $T$ also forms a lattice and a semigroup. Holland [7] has shown that $\mathbf{L} \subseteq \mathbf{T}$. As both the meet and multiplication operations are identical in $\mathbf{L}$ and $\mathbf{T}$, it is natural to ask whether $\mathbf{L}$ forms a sublattice of $T$. In (4.3) and (4.4) it is shown that this is not the case and that in general the join operations of $\mathbf{L}$ and $\mathbf{T}$ do not yield the same results.

2. The lattice-ordered semigroup $L$. A variety of l-groups is the class of all l-groups for which a given set of words (involving the group and lattice operations) are laws. Equivalently a variety of $l$-groups is a class of $l$-groups which is closed with respect to $l$-subgroups, $l$-homomorphic images, and cardinal products (direct products with componentwise ordering). Some of the l-group varieties of particular interest are $\mathcal{E}$ the trivial variety, $\mathcal{Q}$ the abelian variety, $\Re$ the representable variety, $\Re$ the normal-valued variety, and $\mathcal{L}$ the variety of all $l$-groups.

The set $\mathbf{L}$ of all $l$-group varieties is known to be a dually-Brouwer and hence distributive lattice, with intersection functioning as the meet operation, and the join of a collection of varieties defined to be the intersection of all varieties containing those in the collection. Similarly, it is possible to describe the variety generated by an $l$-group $G, l-\operatorname{var}(G)$, as the intersection of all $l$-group varieties containing $G$. In addition, we may define an associative operation on $\mathbf{L}$ such that for $\mathcal{U}, \mathfrak{V} \in \mathbf{L}$, $\mathcal{U} \mathfrak{V}$ contains all $l$-groups $G$ having an $l$-ideal in $\mathcal{U}$ whose quotient lies in $\mathscr{V}$. This operation is compatible with the set containment ordering on $\mathbf{L}$ and distributes over all meets and finite joins from the left.

Martinez [10] has shown that $G \in \mathcal{V}_{1} \vee \mathcal{V}_{2}$ iff $G$ is a subdirect product of l-groups from $\Upsilon_{1}$ and $\Upsilon_{2}$. He has also shown that $\Re$ is idempotent in $L$, i.e. $\Re \Re=\Re$. It is now to be shown that $\Re$ is finitely join-irreducible as well; however, the proof of this fact will require the use of wreath products.

Let $G$ and $H$ be $l$-groups. Due to a theorem of Holland [5], there exist chains $\Omega_{1}$ and $\Omega_{2}$ such that $G$ and $H$ can be considered as $l$-groups of order-preserving permutations ( $l$-permutation groups) on $\Omega_{1}$ and $\Omega_{2}$ respectively. It should be noted that an order-preserving permutation $g$ on a chain $\Gamma$ is considered positive if $\gamma g>\gamma$ for all $\gamma \in \Gamma$. Using this representation for $G$ and $H$, let $(W, \Omega)=$ $\left(G, \Omega_{1}\right) \mathrm{Wr}\left(H, \Omega_{2}\right)$ be the order-preserving permutation group on $\Omega=\Omega_{1} \overline{\times} \Omega_{2}$, where all elements of $W$ are of the form $(\theta, h)$ for $h \in H$ and $\theta: \Omega_{2} \rightarrow G$, and the action of $W$ on $\Omega$ defined by $\left(\alpha_{1}, \alpha_{2}\right)(\theta, h)=\left(\alpha_{1} \theta\left(\alpha_{2}\right), \alpha_{2} h\right)$ for any $\left(\alpha_{1}, \alpha_{2}\right) \in \Omega$. It can be verified that the group operation on $W$ is such that $(\theta, h)(\phi, k)=(\psi, h k)$, where for $\alpha \in \Omega_{2}, \psi(\alpha)=\theta(\alpha) \phi(\alpha h)$. Using this multiplication on $W$ and the usual ordering of order-preserving permutations discussed above, $W$ is an l-group [8], and is called the Wreath product of $G$ with $H$. Within $W$,

$$
W^{\prime}=\left\{(\theta, h) \mid \theta(\alpha)=e_{G} \text { for all but a finite subset of } \Omega_{2}\right\}
$$

forms an $l$-subgroup which also acts as an $l$-permutation group on $\Omega=\Omega_{1} \overline{\times} \Omega_{2}$. This $l$-subgroup is called the wreath product of $G$ with $H$ and is denoted by $\left(W^{\prime}, \Omega\right)=\left(G, \Omega_{1}\right) \mathrm{wr}\left(H, \Omega_{2}\right)$. When an $l$-group $G$ is itself totally ordered, it is 
possible to consider $G$ as an $l$-permutation group on itself, using right translation as the action. In such a case, $G$ is usually preferred over the more cumbersome notation $(G, G)$; so the notation $\left(G, \Omega_{1}\right) \mathrm{Wr} H, G \mathrm{Wr}\left(H, \Omega_{2}\right), G \mathrm{Wr} H$, etc. make sense in this context and will be used where appropriate.

2.1. Lemma. $\mathcal{V} \in \mathbf{L}$ is finitely join-irreducible if $\mathcal{V}$ is closed under wreath (Wreath) products.

Proof. By way of contradiction, suppose $\mathcal{V}$ is finitely join-reducible. Then there exist $\mathscr{U}_{1}, \mathscr{Q}_{2} \in \mathbf{L}$ such that $\mathscr{U}_{1} \subset \mathcal{V}, \mathscr{\vartheta}_{2} \subset \mathcal{V}$ and $\mathcal{V}=\mathscr{U}_{1} \vee \mathcal{U}_{2}$. Since a variety is generated by its subdirectly irreducible $l$-groups [4], there must exist subdirectly irreducible l-groups $H \in \mathcal{V} \backslash \mathcal{Q}_{1}$ and $K \in \mathcal{V} \backslash \mathcal{Q}_{2}$. Holland [5] has shown that there exist chains $\Omega_{1}$ and $\Omega_{2}$ such that $\left(H, \Omega_{1}\right)$ and $\left(K, \Omega_{2}\right)$ are transitive l-permutation groups, i.e. a group $G$ acts transitively on a set $\Gamma$ if for all $\gamma, \delta \in \Gamma$ there exists $g \in G$ such that $\gamma g=\delta$. Then $(W, \Omega)=\left(H, \Omega_{1}\right)$ wr $\left(K, \Omega_{2}\right)$ is also a transitive $l$-permutation group (similarly for the Wreath product), and $W \in \mathcal{V}$ by assumption. Thus, $W \in \mathcal{Q}_{1} \vee \mathcal{U}_{2}$, so $W$ must be a subdirect product of $l$-groups from $\mho_{1}$ and $\mathscr{Q}_{2}$, i.e. there must exist $l$-ideals $A_{1}$ and $A_{2}$ in $W$ such that $A_{1} \cap A_{2}=\{e\}$, $W / A_{1} \in \mathcal{Q}_{1}$, and $W / A_{2} \in \mathcal{Q}_{2}$. If $A_{1}$ were trivial, then $W \in \mathcal{Q}_{1}$; and since $H$ is $l$-embeddable in $W$, then $H \in \mathcal{Q}_{1}$, contradicting our selection of $H$. If $A_{2}$ were trivial, then $W \in \mathscr{Q}_{2}$; and since $K$ is an $l$-homomorphic image of $W, K \in \mathscr{Q}_{2}$, contradicting our selection of $K$. Thus, neither $A_{1}$ nor $A_{2}$ can be trivial. Hence, there must exist $h_{1} \in A_{1}^{+}, h_{2} \in A_{2}^{+}$, and $\alpha, \beta \in \Omega$ such that $\alpha h_{1}>\alpha$ and $\beta h_{2}>\beta$. Also, by transitivity, there exists $g \in W$ such that $\alpha g=\beta$. Now since $\beta g^{-1} h_{1} g=$ $\alpha h_{1} g>\alpha g=\beta$, both $g^{-1} h_{1} g \in A_{1}$ and $h_{2} \in A_{2}$ move $\beta$. But this is impossible for disjoint $l$-ideals. Therefore, $\mathcal{T}$ must be finitely join-irreducible.

\subsection{Theorem. $\Re$ is finitely join-irreducible in $\mathbf{L}$.}

Proof. Martinez [10] has shown that $\Re$ is idempotent, and hence closed with respect to all extensions including Wreath and wreath products. Thus, by (2.1) $\Re$ is finitely join-irreducible.

3. Properties of the Scrimger varieties. It is known that $Q$ is the smallest nontrivial variety in $\mathbf{L}$. In an attempt to generalize $\mathcal{Q}$, the family of varieties $\mathcal{L}_{n}$ for $n \in N$ were introduced, where $\mathscr{L}_{n}$ is the variety of all $l$-groups in which all $n$th powers commute. Obviously, $\mathscr{L}_{1}=\mathbb{Q}$ and $\mathfrak{L}_{m} \subseteq \mathfrak{L}_{n}$ if $m \mid n$. Also, within each $\mathfrak{L}_{n}$ is an $l$-group [9] of the form

$$
G_{n}=\{(\phi, k) \in Z \operatorname{Wr} Z \mid \phi(i)=\phi(j) \text { if } i \equiv j(\bmod n)\} .
$$

Such an l-group may also be viewed as a lexicographically-ordered splitting extension by $Z$ of a cardinal product of $n$ copies of $Z$, with the multiplication defined by

$$
\begin{aligned}
\left(\left(r_{0}, \ldots, r_{n-1}\right), k\right) & \left(\left(s_{0}, \ldots, s_{n-1}\right), l\right) \\
= & \left(\left(r_{0}+s_{0+k}, \ldots, r_{n-1}+s_{n-1+k}\right), k+l\right),
\end{aligned}
$$


where $r_{i}, s_{j}, k, l \in Z$ and all subscripts are considered $\bmod n$. These $l$-groups and the varieties they generate, where $\delta_{n}=l-\operatorname{var}\left(G_{n}\right)$ is known as the $n$th Scrimger variety, have been shown to be of great interest in the study of $L$. Some of the more useful facts concerning the $G_{n}$ and $\delta_{n}$ are:

3.1. For any $n \in N, G_{n}$ is an $l$-subgroup of $Z \mathrm{Wr} Z$.

3.2. For any $n \in N, G_{n}$ is a subdirectly irreducible l-group.

3.3. For any $n \in N, G_{n} \in \mathbb{Q}^{2}=\mathscr{Q} \mathbb{Q}$.

3.4. For $m, n \in N, G_{m} \in \mathfrak{L}_{n}$ iff $m \mid n$.

3.5. For $m, n \in N, \mathfrak{L}_{m} \subseteq \mathfrak{L}_{n}$ iff $m \mid n$.

3.6. For $m, n>1, \mathcal{S}_{m} \vee \mathcal{S}_{n} \subset \mathcal{S}_{m n}$ and $\mathfrak{L}_{m} \vee \mathfrak{L}_{n} \subset \mathfrak{L}_{m n}$.

3.7. For any $n \in N, \mathcal{R} \wedge \mathcal{L}_{n}=\mathbb{Q}$ and $\mathscr{R} \wedge \mathcal{S}_{n}=\mathbb{Q}$ [9].

3.8. For $m, n \in N$ such that $(m, n)=1, \mathfrak{L}_{m} \wedge \mathfrak{L}_{n}=\mathbb{Q}, \delta_{m} \wedge \delta_{n}=\mathbb{Q}$, and $\mathcal{L}_{m} \wedge \delta_{n}=\mathscr{Q}[13]$.

3.9. For any prime $p, \delta_{p}$ covers $\mathscr{Q}$ in $\mathbf{L}$, i.e. no variety lies between $\mathscr{Q}$ and $\delta_{p}$ [13].

3.10. Proposition. For any variety $\mathfrak{V} \in \mathbf{L}$ and any prime $p$ such that $\mathfrak{V} \wedge \delta_{p}=$ $\mathcal{Q}, \mathfrak{V} \vee \mathcal{S}_{p}$ covers $\mathfrak{T}$ in $\mathbf{L}$.

Proof. Since $\mathbf{L}$ is a distributive lattice, the interval $\{\mathscr{W} \in \mathbf{L} \mid \mathcal{V} \subset \mathcal{W} \subset \mathcal{V} \vee$

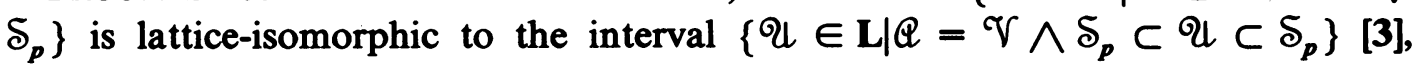
and hence is empty.

Thus, it is known that for any prime $p, \Re \vee \mathscr{S}_{p}$ covers $\Re$. Also, for any integer $n$ and prime $p$ such that $p \nmid n, \delta_{n} \vee \delta_{p}$ covers $\delta_{n}$ and $\mathcal{E}_{n} \vee \delta_{p}$ covers $\mathcal{L}_{n}$. Extending this notion by means of induction leads us to the following result.

3.11. Corollary. For any finite set of distinct primes $\left\{p_{1}, p_{2}, \ldots, p_{m}\right\}, \bigvee\left\{\delta_{p_{i}} \mid i\right.$ $=1, \ldots, m\}$ covers $\bigvee\left\{\delta_{p_{i}} \mid i=1, \ldots, m-1\right\}$.

3.12. Proposition. For any primes $p_{1}, \ldots, p_{m}, q_{1}, \ldots, q_{n}$,

$$
\bigvee\left\{\delta_{p_{i}} \mid i=1, \ldots, m\right\} \subseteq \bigvee\left\{\delta_{q} \mid j=1, \ldots, n\right\}
$$

iff $\left\{p_{1}, \ldots, p_{m}\right\} \subseteq\left\{q_{1}, \ldots, q_{n}\right\}$.

Proof. Sufficiency is obvious. To prove necessity, suppose $\bigvee\left\{\delta_{p_{t}} \mid i=\right.$ $1, \ldots, m\} \subseteq \bigvee\left\{\delta_{q} \mid j=1, \ldots, n\right\}$. Then for any $p_{i}$,

$$
\delta_{p_{i}} \subseteq \delta_{p_{i}} \wedge\left(\vee\left\{\delta_{q_{j}} \mid j=1, \ldots, n\right\}\right)=\bigvee\left\{\left(\delta_{p_{t}} \wedge \delta_{q_{j}}\right) \mid j=1, \ldots, n\right\}
$$

Hence, for some $j, \delta_{p_{i}}=\delta_{p_{i}} \wedge \delta_{q_{q}}$, implying that $p_{i}=q_{j}$.

Accordingly, the sublattice of $\mathbf{L}$ generated by $\left\{\delta_{p} \mid p\right.$ is prime $\}$ is lattice-isomorphic to the lattice of finite sets of primes. To investigate whether or not the complete sublattice of $\mathbf{L}$ generated by $\left\{\delta_{p} \mid p\right.$ is prime $\}$ is lattice-isomorphic to the full Boolean lattice of sets of primes, it is helpful to consider conditions under which an $l$-group is $l$-isomorphic to $G_{n}$ for some $n \in N$.

3.13. TheOREM. An l-group $G$ is l-isomorphic to $G_{n}$ iff it is generated (as a group) by two positive elements $a$ and $b$ such that

(i) $a<b$, and

(ii) for $1<i<n-1, b^{-i} a b^{i} \wedge a=e$ and $b^{-n} a b^{n}=a$. 
Proor. To prove necessity, it need only be noted that the elements $((1,0, \ldots, 0), 0)$ and $((0, \ldots, 0), 1)$ of $S_{n}$ satisfy the conditions on $a$ and $b$ respectively.

To prove sufficiency, let us assume that $G$ is an $l$-group generated by positive elements $a$ and $b$ satisfying conditions (i) and (ii). Define a function $\nu: G_{n} \rightarrow G$ by:

$$
\left(\left(r_{0}, \ldots, r_{n-1}\right), k\right) \nu=\left(\prod_{i=0}^{n-1}\left(b^{-i} a b^{i}\right)^{r_{i}}\right) b^{k},
$$

where $r_{i}, k \in Z$. To verify that $\nu$ preserves the group operations, it should be noted that condition (ii) guarantees that all conjugates of $a$ by powers of $b$ commute in $G$. So, for arbitrary elements of $G_{n}$,

$$
\begin{aligned}
{\left[\left(\left(r_{0}, \ldots, r_{n-1}\right), k\right)\right.} & \left.\left(\left(s_{0}, \ldots, s_{n-1}\right), l\right)\right] \nu \\
= & {\left[\left(\left(r_{0}+s_{0+k}, \ldots, r_{n-1}+s_{n-1+k}\right), k+l\right)\right] \nu } \\
& =\left(\prod_{i=0}^{n-1}\left(b^{-i} a b^{i}\right)^{r_{1}+s_{i+k}}\right) b^{k+l},
\end{aligned}
$$

while

$$
\begin{aligned}
\left(\left(r_{0}, \ldots, r_{n-1}\right), k\right) \nu\left(\left(s_{0}, \ldots, s_{n-1}\right), l\right) \nu \\
=\left(\prod_{i=0}^{n-1}\left(b^{-i} a b^{i}\right)^{r_{i}}\right) b^{k}\left(\prod_{j=0}^{n-1}\left(b^{-j} a b^{j}\right)^{s_{j}}\right) b^{l} \\
=\left(\prod_{i=0}^{n-1}\left(b^{-i} a b^{i}\right)^{r_{i}}\right)\left(\prod_{j=0}^{n-1}\left(b^{-j+k} a b^{j-k}\right)^{s_{j}}\right) b^{k+l} \\
=\left(\prod_{i=0}^{n-1}\left(b^{-i} a b^{i}\right)^{r_{i}}\right)\left(\prod_{i=0}^{n-1}\left(b^{-i} a b^{i}\right)^{s_{i+k}}\right) b^{k+l} \\
=\left(\prod_{i=0}^{n-1}\left(b^{-i} a b^{i}\right)^{r_{i}+s_{i+k}}\right) b^{k+l},
\end{aligned}
$$

with all subscripts considered $\bmod n$. Hence, $\nu$ is a group homomorphism.

In order to prove that $\nu$ preserves the lattice operations as well, it is helpful to first show that $b$ exceeds the $l$-ideal of $G$ generated by $a$. Suppose there exists $m \in N$ such that $a^{m} \nless b$. If $a^{m}=b$, then $a$ and $b$ would commute. So, in fact, $a^{m} \nless b$, and then $\left(b^{-1} a b\right)^{m} \nless b$. By Holland's theorem [5], there exists a chain $\Gamma$ such that $G$ acts as an $l$-group of order-preserving permutations on $\Gamma$. Thus, there exists some $\gamma \in \Gamma$ for which

$$
\gamma\left(b^{-1} a b\right)^{m}>\gamma b>\gamma .
$$

Since $\gamma$ is moved by $\left(b^{-1} a b\right)^{m}$, it is moved by $b^{-1} a b$. Then condition (ii) implies that $\gamma a=\gamma$, so $(\gamma b)\left(b^{-1} a b\right)=\gamma b$ and $(\gamma b)\left(b^{-1} a b\right)^{m}=\gamma b$. But by $(*),(\gamma b)\left(b^{-1} a b\right)^{m}$ $>\gamma\left(b^{-1} a b\right)^{m}>\gamma b$. Thus $a^{m}<b$ for all $m \in Z$, and similarly $\left(b^{-i} a b^{i}\right)^{m}<b$ for all $i, m \in Z$. Now this and the disjointness of the conjugates leads us to:

$$
\prod_{i=0}^{n-1}\left(b^{-i} a b^{i}\right)^{r_{i}}=\bigvee_{i=0}^{n-1}\left(b^{-i} a b^{i}\right)^{r_{i}}<b
$$


for any $r_{0}, \ldots, r_{n-1} \in Z$. Again this inequality must in fact be proper, as $a$ and $b$ do not commute in $G$. Hence, $b$ exceeds the $l$-ideal of $G$ generated by $a$ and so dominates the order on $G$.

Now for any $\left(\left(r_{0}, \ldots, r_{n-1}\right), k\right) \in G_{n},\left(\left(r_{0}, \ldots, r_{n-1}\right), k\right) \vee((0, \ldots, 0), 0)$ is computed to be $\left(\left(r_{0}, \ldots, r_{n-1}\right), k\right)$ if $k>0,((0, \ldots, 0), 0)$ if $k<0$, and $\left(\left(r_{0} \vee\right.\right.$ $\left.\left.0, \ldots, r_{n-1} \vee 0\right), 0\right)$ if $k=0$. In the case where $k>0$,

$$
\left(\prod_{i=0}^{n-1}\left(b^{-i} a b^{i}\right)^{r_{i}}\right) b^{k} \vee e=\left(\prod_{i=0}^{n-1}\left(b^{-i} a b^{i}\right)^{r_{i}}\right) b^{k}
$$

since $b^{k}$ dominates. Similarly, for $k<0$,

$$
\left(\prod_{i=0}^{n-1}\left(b^{-i} a b^{i}\right)^{r_{i}}\right) b^{k} \vee e=e
$$

For $k=0$, let us divide $\{0, \ldots, n-1\}$ into two disjoint sets $I=\left\{i \mid r_{i}>0\right\}$ and $J=\left\{j \mid r_{j}<0\right\}$. Then

$$
\begin{aligned}
\left(\prod_{i=0}^{n-1}\left(b^{-i} a b^{i}\right)^{r_{i}}\right) \vee e & =\left[\prod_{i \in I}\left(b^{-i} a b^{i}\right)^{r_{i}} \vee \prod_{j \in J}\left(b^{-j} a b^{j}\right)^{-r_{j}}\right] \prod_{j \in J}\left(b^{-j} a b^{j}\right)^{r_{j}} \\
& =\left[\prod_{i \in I}\left(b^{-i} a b^{i}\right)^{r_{i}} \prod_{j \in J}\left(b^{-j} a b^{j}\right)^{-r_{j}}\right] \prod_{j \in J}\left(b^{-j} a b^{j}\right)^{r_{j}} \\
& =\prod_{i \in I}\left(b^{-i} a b^{i}\right)^{r_{i}}=\prod_{i=0}^{n-1}\left(b^{-i} a b^{i}\right)^{r_{i} \vee 0} .
\end{aligned}
$$

Thus $\nu$ is an $l$-homomorphism.

Suppose $\left(\Pi_{i=0}^{n-1}\left(b^{-i} a b^{i}\right)^{r_{i}}\right) b^{k}=e$. Then $k=0$, since $b$ strictly exceeds all products of conjugates of $a$. Now let us divide $\{0, \ldots, n-1\}$ into $I$ and $J$ as above. Then $\Pi_{i \in I}\left(b^{-i} a b^{i}\right)^{r_{i}}=\Pi_{j \in J}\left(b^{-j} a b^{j}\right)^{-r_{j}}$. So if some $r_{i}>0$, then

$$
\begin{aligned}
\left(b^{-i} a b^{i}\right)^{r_{i}} & =\left(b^{-i} a b^{i}\right)^{r_{i}} \wedge \prod_{j \in J}\left(b^{-j} a b^{j}\right)^{-r_{j}} \\
& =\prod_{j \in J}\left(\left(b^{-i} a b^{i}\right)^{r_{i}} \wedge\left(b^{-j} a b^{j}\right)^{-r_{j}}\right)=e,
\end{aligned}
$$

contradicting the fact that $b^{-i} a b^{i}>e$. Thus $r_{0}=r_{1}=\ldots=r_{n-1}=0$ and $\nu$ is one-to-one.

To complete the proof it is necessary only to note that since every element of $G$ is of the form $a^{m_{0}} b^{m_{1}} a^{m_{2}} \ldots b^{m_{q}}$, such a term can be re-expressed as the image of some element of $G_{n}$ by merely adding and subtracting appropriate powers of $b$ throughout.

3.14. THEOREM. If $\left\{m_{\alpha} \mid \alpha \in N\right\}$ is any infinite subset of $N$, then $\bigvee\left\{\delta_{m_{m}} \mid \alpha \in N\right\}$ $=\bigvee\left\{\delta_{n} \mid n \in N\right\}$, i.e. there is only one variety in $\mathbf{L}$ that is an infinite join of $\mathcal{S}_{n}$ 's.

Proof. It is sufficient to show that for $n \in N, G_{n} \in \bigvee\left\{\delta_{m_{n}} \mid \alpha \in N\right\}$. If $n \mid m_{\beta}$ for some $\beta$, then $\delta_{n} \subseteq \delta_{m_{\beta}} \subseteq V\left\{\delta_{m_{\alpha}} \mid \alpha \in N\right\}$; therefore, we may assume that for all $m_{\alpha}, n \nmid m_{\alpha}$. We may also assume that $n<m_{\alpha}$ for all $\alpha \in N$, since there is only a finite set for which this is not the case, and that the set $\left\{\delta_{m_{\alpha}}\right\}$ is ordered so that $m_{\alpha}<m_{\alpha+1}$ for all $\alpha \in N$. 
Now let $G$ be the cardinal product of the $G_{m_{a}}$ 's. So elements of $G$ are of the form $\left(\left(\phi_{1}, k_{1}\right),\left(\phi_{2}, k_{2}\right),\left(\phi_{3}, k_{3}\right), \ldots\right)$, where for all $\alpha \in N, k_{\alpha} \in Z$ and $\phi_{\alpha}: Z \rightarrow Z$ such that $\phi_{\alpha}\left(j+m_{\alpha}\right)=\phi_{\alpha}(j)$ for every $j \in Z$, and the $k_{\alpha}$ dominate the order.

Let us pick $a, b \in G$ of the form $a=\left(\left(\psi_{1}, 0\right),\left(\psi_{2}, 0\right),\left(\psi_{3}, 0\right), \ldots\right)$ and $b=$ $((0,1),(0,1),(0,1), \ldots)$ where for all $\alpha \in N, \psi_{\alpha}(j)$ is 1 if $j \equiv 0(\bmod n)$ and is 0 otherwise and $O(j)=0$ for $0<j<m_{\alpha}$ and extended $\bmod m_{\alpha}$ to all $j \in Z$. Then certainly $b>a>e$ in $G$. For $1<i<n-1$,

$$
b^{-i} a b^{i}=\left(\left(\psi_{1, i}, 0\right),\left(\psi_{2, i}, 0\right),\left(\psi_{3, i}, 0\right), \ldots\right),
$$

where $\psi_{\alpha, i}(j)=\psi_{\alpha}(j-i)$ for all $\alpha \in N$ and $j \in Z$. Therefore,

$$
b^{-i} a b^{i} \wedge a=\left(\left(\psi_{1, i} \wedge \psi_{1}, 0\right),\left(\psi_{2, i} \wedge \psi_{2}, 0\right)\left(\psi_{3, i} \wedge \psi_{3}, 0\right), \ldots\right),
$$

where $\left(\psi_{\alpha, i} \wedge \psi_{\alpha}\right)(j)=0$ for all $\alpha \in N$ and $0<j<m_{\alpha}-1$ and $\left(\psi_{\alpha, i} \wedge \psi_{\alpha}\right)(0)$ is 1 if $i \equiv m_{\alpha}(\bmod n)$ and is 0 otherwise. Similarly,

$$
b^{-n} a b^{n}=\left(\left(\psi_{1, n}, 0\right),\left(\psi_{2, n}, 0\right),\left(\psi_{3, n}, 0\right), \ldots\right)
$$

with $\psi_{\alpha, n}(j)=\psi_{\alpha}(j-n)$ for all $\alpha \in N$ and $j \in Z$. Hence,

$$
b^{-n} a b^{n} a^{-1}=\left(\left(\psi_{1, n}-\psi_{1}, 0\right),\left(\psi_{2, n}-\psi_{2}, 0\right),\left(\psi_{3, n}-\psi_{3}, 0\right), \ldots\right),
$$

where for all $\alpha \in N,\left(\psi_{\alpha, n}-\psi_{\alpha}\right)(0)=\psi_{\alpha}(0-n)-\psi_{\alpha}(0)=\psi_{\alpha}\left(m_{\alpha}-n\right)-\psi_{\alpha}(0)=$ $0-1=-1$ since $n<m_{\alpha}$ and $n \nmid m_{\alpha} ;\left(\psi_{\alpha, n}-\psi_{\alpha}\right)(j)$ is 1 if $m_{\alpha} \equiv(n-j)(\bmod n)$ and is 0 otherwise for $0<j<n-1$; and $\left(\psi_{\alpha, n}-\psi_{\alpha}\right)(j)=0$ for $n<j<m_{\alpha}-1$. So for any $\alpha \in N,\left(\psi_{\alpha, n}-\psi_{\alpha}\right)$ has exactly two nonzero entries, both of which occur within the first $n$ terms.

Now, let $M$ be the $l$-ideal of $G$ generated by $b^{-n} a b^{n} a^{-1}$ and $\left\{b^{-i} a b^{i} \wedge a \mid 1<i\right.$ $\langle n-1\}$. Obviously, $b \notin M$. Also, for any $g \in M$, where $g=\left(\left(\phi_{1}, 0\right),\left(\phi_{2}, 0\right)\right.$, $\left.\left(\phi_{3}, 0\right), \ldots\right)$, there is a uniform bound on the number of nonzero entries for the $\phi_{\alpha}$. However, this is not true for the $\psi_{\alpha}$, so $a \notin M$. Obviously, $a M$ and $b M$ in $G / M$ satisfy the conditions of (3.13), so the $l$-subgroup of $G / M$ generated by $a M$ and $b M$ is $l$-isomorphic to $G_{n}$. Thus, $G_{n} \in \bigvee\left\{\delta_{m_{n}} \mid \alpha \in N\right\}$.

Using this theorem, it is now possible to answer a question posed by Martinez [10].

\subsection{COROllaRY. $\mathrm{L}$ is not a Brouwer lattice.}

Proof. $\delta_{3}=\delta_{3} \wedge\left(\bigvee\left\{\delta_{2^{n}} \mid n \in N\right\}\right)$ according to (3.14), but $\bigvee\left\{\left(\delta_{3} \wedge \delta_{2^{n}}\right) \mid n \in\right.$ $N\}=\mathbb{Q}$ by (3.8).

Note that the proof above is actually of a stronger result, i.e. meets do not distribute over infinite joins even of towers in L. Also (3.14) eliminates the possibility of dropping the idea of taking quotients when generating an infinite join of varieties.

Since for all $n \in N, G_{n}$ is an $l$-subgroup of $Z \mathrm{Wr} Z, \bigvee\left\{\delta_{n} \mid n \in N\right\} \subseteq l$ $\operatorname{var}(Z \mathrm{Wr} Z)$. In order to determine whether this containment is proper, it is necessary to look at a generalization of (3.13). 
3.16. Theorem. An l-group $G$ is l-isomorphic to $Z$ wr $Z$ iff it is generated (as a group) by two positive elements $a$ and $b$ such that

(i) $a<b$, and

(ii) $b^{-i} a b^{i} \wedge a=e$, for all $i \in N$.

Proof. To prove necessity, the elements $a=(\theta, 0)$ and $b=(0,1)$ of $Z$ wr $Z$, where $\theta(j)$ is 1 if $j=0$ and is 0 otherwise and $\theta(j)=0$ for all $j \in Z$, can readily be shown to generate $Z$ wr $Z$ and satisfy conditions (i) and (ii).

For the proof of sufficiency, define $\nu: Z$ wr $Z \rightarrow G$ by $(\phi, k) \nu=$ $\left(\Pi_{i \in Z}\left(b^{-i} a b^{i}\right)^{\phi(i)}\right) b^{k}$. Since for only a finite number of integers $i$ is $\phi(i) \neq 0$, this function is well defined. By arguments analogous to those of (3.13), it can be shown that $\nu$ is an $l$-isomorphism.

3.17. ThEOREM. $\bigvee\left\{\delta_{n} \mid n \in N\right\}=l$-var( $Z$ Wr $\left.Z\right)=l$-var( $Z$ wr $\left.Z\right)$.

Proof. First it should be noted that $Z \mathrm{Wr} Z$ is an $\mathcal{L}$-extension of $Z$ wr $Z$, i.e. every element of $Z \mathrm{Wr} Z$ can be expressed as a join of a pairwise disjoint subset of elements of $Z$ wr $Z$. As Bernau [1] has shown that $l$-group varieties are closed under $\mathcal{L}$-extensions, it suffices simply to show that $Z$ wr $Z \in$ $\bigvee\left\{\delta_{n} \mid n \in N\right\}$.

Let $G$ be the cardinal product of the $G_{n}$. Then elements of $G$ can be expressed in the form $\left(\left(\phi_{1}, k_{1}\right),\left(\phi_{2}, k_{2}\right),\left(\phi_{3}, k_{3}\right), \ldots\right)$, where for every $n \in N, k_{n} \in Z$ and $\phi_{n}$ : $Z \rightarrow Z$ such that $\phi_{n}(j+n)=\phi_{n}(j)$ for all $j \in Z$, and where the $k_{n}$ dominate the order in $G$. Let us pick $a, b \in G$ of the form $a=\left(\left(\psi_{1}, 0\right),\left(\psi_{2}, 0\right),\left(\psi_{3}, 0\right), \ldots\right)$ and $b=((0,1),(0,1), \ldots)$ where for all $n \in N, \psi_{n}(j)$ is 1 if $j \equiv 0(\bmod n)$ and is 0 otherwise and $0(j)=0$ for all $j \in Z$. Thus, $b>a>e$.

For any $i \in N, b^{-i} a b^{i}=\left(\left(\psi_{1, i}, 0\right),\left(\psi_{2, i}, 0\right),\left(\psi_{3, i}, 0\right), \ldots\right)$ where $\psi_{n, i}(j)=\psi_{n}(j-$ $i)$ for all $n \in N$ and $j \in Z$. Therefore,

$$
b^{-i} a b^{i} \wedge a=\left(\left(\psi_{1, i} \wedge \psi_{1}, 0\right),\left(\psi_{2, i} \wedge \psi_{2}, 0\right),\left(\psi_{3, i} \wedge \psi_{3}, 0\right), \ldots\right)
$$

where for any $n \in N,\left(\psi_{n, i} \wedge \psi_{n}\right)(j)=1$ if both $i, j \equiv 0(\bmod n)$ and is 0 otherwise. So for any given $i$, only a finite number of $n$ 's will divide $i$; thus, only a finite number of the $\left(\psi_{n, i} \wedge \psi_{n}\right)$ will be nonzero functions for that $i$.

Let $M$ be the $l$-ideal of $G$ generated by $\left\{b^{-i} a b^{i} \wedge a \mid i \in N\right\}$. Obviously, $b \notin M$; and since all of the $\psi_{n}$ are nonzero functions, $a \notin M$. Hence the $l$-subgroup of $G / M$ generated by $a M$ and $b M$ is $l$-isomorphic to $Z$ wr $Z$ by (3.16), and $Z$ wr $Z$ $\in V\left\{\delta_{n} \mid n \in N\right\}$.

4. Varieties and torsion classes. An alternate system of classification of $l$-groups is that of torsion classes [11]. A torsion class is a collection of $l$-groups that is closed under taking convex $l$-subgroups, $l$-homomorphic images, and joins of convex $l$-subgroups in the collection. Let $\mathbf{T}$ denote the class of all torsion classes of l-groups. Then using definitions analogous to those for varieties, it is possible to define the operations of meet, join, and multiplication on $\mathbf{T}$. It is also worthy of note that for any $l$-group $G$, the join of all convex $l$-subgroups of $G$ belonging to a torsion class $\mathfrak{T}$ is itself in $\mathcal{T}$. This join is called the $\mathfrak{T}$-torsion radical of $G$, denoted $\mathcal{T}(G)$, and is a characteristic $l$-ideal of $G$. It follows that $G \in \mathcal{T}$ iff $G=\mathcal{T}(G)$, and $G \in \mathcal{T} \vee U$ iff $G=\mathcal{T}(G)+\mathcal{U}(G)$ for any $\mathcal{T}, \mathcal{U} \in \mathbf{T}$. 
Holland has shown that all $l$-group varieties are torsion classes [7], and Martinez has shown that $T$ is a complete Brouwer lattice [11]. Hence, by (3.15) we have that $\mathbf{L}$ is not a complete sublattice of $\mathbf{T}$, as otherwise it would inherit the Brouwerian structure. It then remains to ask whether $\mathbf{L}$ is even a sublattice of $T$.

4.1. Definition. A variety $\mathcal{V}$ is said to have the lex property if for every $G \in \mathcal{V}$, $G_{\sharp} \in \mathcal{V}$, where $G_{\sharp}=G \overleftarrow{\times} Z$.

4.2. Proposition. If $\left\{\mathfrak{V}_{\alpha} \mid \alpha \in \mathfrak{U}\right\}$ is a collection of varieties having the lex property and $\mathcal{Q} \in \mathbf{L}$, then:

(i) $\wedge \mathfrak{V}_{\alpha}$ and $\bigvee \mathfrak{V}_{\alpha}$ have the lex property,

(ii) $\mathcal{Q} \mathfrak{V}_{\alpha}$ has the lex property.

Proof. (i) That $\wedge \mathfrak{V}_{\alpha}$ has the lex property is obvious. If $G \in \bigvee \mathfrak{V}_{\alpha}$, then $G \approx H / K$ where $H$ has $l$-ideals $N_{\alpha}$ with $\cap N_{\alpha}=\{e\}$ and $H / N_{\alpha} \in \mathscr{V}_{\alpha}$ for all $\alpha \in \mathfrak{A}$. Then $G_{\sharp} \approx(H / K)_{\sharp} \approx H_{\sharp} /(K \times\{0\})$, where $N_{\alpha} \times\{0\}$ is an l-ideal of $H_{\sharp}$ with $\cap\left(N_{\alpha} \times\{0\}\right)=\{(e, 0)\}$ and $H_{\sharp} /\left(N_{\alpha} \times\{0\}\right) \approx\left(H / N_{\alpha}\right)_{\sharp} \in \mathfrak{V}_{\alpha}$, since $\mathfrak{V}_{\alpha}$ has the lex property. Thus, $G_{\sharp} \in \vee \mathfrak{V}_{\alpha}$.

(ii) If $G \in \mathcal{Q} \mathfrak{V}_{\alpha}$, then $G$ has an $l$-ideal $N \in \mathcal{Q}$ with $G / N \in \mathscr{V}_{\alpha}$. Therefore, $N \times\{0\}$ is an $l$-ideal of $G_{\sharp}$ with $G_{\sharp} /(N \times\{0\}) \approx(G / N)_{\sharp} \in \mathcal{V}_{\alpha}$ since $\mathfrak{V}_{\alpha}$ has the lex property. Hence, $G_{\sharp} \in \mathcal{U} \mathfrak{V}_{\alpha}$.

4.3. Theorem. Let $\left\{\mathfrak{V}_{\alpha} \mid \alpha \in \mathfrak{A}\right\}$ be a collection of varieties, all having the lex property, such that $\mathfrak{V}_{\beta} \subset \mathbf{L} \bigvee\left\{\mathfrak{V}_{\alpha} \mid \alpha \in \mathfrak{A}\right\}$ for all $\beta \in \mathfrak{A}$. Then $\mathbf{L} \bigvee \mathfrak{V}_{\alpha} \supset{ }_{\mathbf{T}} \bigvee \mathfrak{V}_{\alpha}$ (where $\mathrm{L} V$ and $\mathrm{T} V$ denote joins in $\mathrm{L}$ and $\mathrm{T}$ respectively).

Proof. Since $\mathbf{L} \subseteq \mathbf{T}, \mathbf{L} \vee \mathfrak{V}_{\alpha} \supseteq T_{\mathbf{T}} \bigvee \mathfrak{V}_{\alpha}$. Thus, we need only show the containment to be proper. Without loss of generality, we may assume that $\mathfrak{A}$ is wellordered and that for any $\alpha \in \mathfrak{A}, \mathfrak{V}_{\alpha} \nsubseteq \cup\left\{\mathfrak{V}_{\beta} \mid \beta<\alpha\right\}$; since otherwise, such "unneeded" varieties could be thrown out without affecting the final join in either $\mathbf{L}$ or $\mathbf{T}$.

If $\mathfrak{U}$ has no largest element, then choose $G_{\alpha} \in \mathfrak{V}_{\alpha} \backslash \cup\left\{\mathfrak{V}_{\beta} \mid \beta<\alpha\right\}$, for each $\alpha \in \mathfrak{A}$. Then by (4.2), $G=\left(\Pi G_{\alpha}\right)_{\sharp} \in{ }_{L} \vee \mathscr{V}_{\alpha}$. If $G \in T \bigvee \mathscr{V}_{\alpha}$, then $G=\Sigma \mathscr{V}_{\alpha}(G)$, where $\mathfrak{V}_{\alpha}(G)$ is the $\mathfrak{V}_{\alpha}$-torsion radical of $G$ and hence an $l$-ideal of $G$. All proper $l$-ideals of $G$ are contained within $\left(I I G_{\alpha}\right) \times\{0\}$, and such $l$-ideals cannot generate $G$. Thus, for some $\beta \in \mathfrak{A}, G=\mathfrak{V}_{\beta}(G)$, implying that $G \in \mathfrak{V}_{\beta}$. Since $G_{\beta+1}$ is an l-subgroup of $G$ chosen outside of $\mathcal{V}_{\beta}$, the desired contradiction has been reached.

If $\mathfrak{A}$ has a largest element $\alpha_{0}$, then for some $\gamma \in \mathfrak{A}, \mathfrak{V}_{\gamma} \nsubseteq \mathfrak{V}_{\alpha_{0}}$. So, choose $G_{\alpha} \in \mathfrak{V}_{\alpha} \backslash \cup\left\{\mathfrak{V}_{\beta} \mid \beta<\alpha\right\}$ for each $\alpha \in \mathfrak{A}$ as before, and choose $H_{\gamma} \in \mathfrak{V}_{\gamma} \backslash \mathfrak{V}_{\alpha_{0}}$. Then $G=\left(H_{\gamma} \times \Pi G_{\alpha}\right)_{\sharp} \in_{\mathrm{L}} \bigvee \mathcal{V}_{\alpha}$, and by an argument similar to that above, $G \notin$ $T \bigvee \mathscr{V}_{\alpha}$.

In order to state finally that $L$ is not a sublattice of $T$, it remains to be determined whether or not there exist varieties having the lex property, and if some exist, how prevalent they are in $\mathbf{L}$. First, as a lex product is simply a direct product of the groups involved, any l-group variety definable solely in terms of its group operations will have the lex property. Thus, $Q$ and $\mathscr{L}_{n}(n \in N)$ have the lex property. In fact, all of the varieties to which reference has been made within this paper have the lex property, making $L$ very far indeed from being a sublattice of $T$. 
4.4. Proposition. $\Re, \Re, \mathscr{Q}$ (the weakly abelian variety), and $\mathcal{S}_{n}(n \in N)$ have the lex property.

Proof. (i) For $G \in \mathfrak{T}$, we wish to show that $x_{1}^{2} x_{2}^{2}>x_{2} x_{1}$ for all positive elements $x_{1}, x_{2} \in G_{\sharp}$. Let $x_{1}=(g, k)$ and $x_{2}=(h, l)$ where $g, h \in G$ and $k, l \in$ $Z$. These elements are positive iff $k>0$ or $k=0$ and $g \in G^{+}$and $l>0$ or $l=0$ and $h \in G^{+}$. If either $k>0$ or $l>0$, then $2 k+2 l>l+k$, making

$$
(g, k)^{2}(h, l)^{2}=\left(g^{2} h^{2}, 2 k+2 l\right)>(h g, l+k)=(h, l)(g, k) .
$$

If both $k, l=0$, then $(g, 0)^{2}(h, 0)^{2}=\left(g^{2} h^{2}, 0\right)>(h g, 0)=(h, 0)(g, 0)$ since $G \in$ $\Re$. Thus, $G_{\sharp} \in \Re$.

(ii) For $G \in R$, we wish to show that $x_{1} \wedge\left(x_{2}^{-1} x_{1}^{-1} x_{2}\right)<e$ for all $x_{1}, x_{2} \in G_{\sharp}$. Again letting $x_{1}=(g, k)$ and $x_{2}=(h, l)$, we have that

$$
(g, k) \wedge(h, l)^{-1}(g, k)^{-1}(h, l)=(g, k) \wedge\left(h^{-1} g^{-1} h,-k\right),
$$

which equals $(g, k)$ if $k<0$ and $\left(g \wedge h^{-1} g^{-1} h, 0\right)$ if $k=0$ and $\left(h^{-1} g^{-1} h,-k\right)$ if $k>0$. In all cases, the outcome is less than or equal to $(e, 0)$; so $G_{\sharp} \in R$.

(iii) For $G \in \mathscr{W}$, it is sufficient to show that $x_{1}^{2}>x_{2}^{-1} x_{1} x_{2}$ for any $x_{2}$ and positive $x_{1}$ in $G_{\#}$. Letting $x_{1}=(g, k)$ and $x_{2}=(h, l)$, with either $k>0$ or $k=0$ and $g \in G^{+}$, then clearly $(g, k)^{2}=\left(g^{2}, 2 k\right)>\left(h^{-1} g h, k\right)=(h, l)^{-1}(g, k)(h, l)$. So $G_{\sharp} \in$ ขr.

(iv) To show that $\delta_{n}$ has the lex property, it suffices to show that $\left(G_{n}\right)_{\sharp} \in \delta_{n}$. Let $G=\left(\Pi_{i=1}^{\infty} G_{n}\right) /\left(\sum_{i=1}^{\infty} G_{n}\right)$; then $G \in \mathcal{S}_{n}$. Let $H_{n}$ be the diagonal $l$-subgroup of $\Pi_{i=1}^{\infty} G_{n}$, with $K_{n}$ its canonical image in $G$. Clearly $K_{n}$ and $G_{n}$ are $l$-isomorphic; let us denote this $l$-isomorphism by $\nu$. Let $g \in G$ correspond to $((0, n),(0,2 n)$, $(0,3 n), \ldots) \in \Pi_{i=1}^{\infty} G_{n}$. Then for any $h \in K_{n}, g>h$ and $g h=h g$. Hence, the $l$-subgroup of $G$ generated by $K_{n}$ and $g$ is $l$-isomorphic to $\left(G_{n}\right)_{\sharp}$ via the map which sends $h^{m}{ }^{m}$ to $(h \nu, m)$ for $h \in K_{n}$ and $m \in Z$. So $\left(G_{n}\right)_{\sharp} \in \mathcal{S}_{n}$.

\section{BiBLIOGRAPHY}

1. S. J. Bernau, Varieties of lattice groups are closed under $\mathfrak{L}$-completion, Sympos. Math. 21 (1977).

2. G. Birkhoff, Lattice theory, Amer. Math. Soc. Colloq. Publ., vol. 25, Amer. Math. Soc., Providence, R. I., 1940.

3. G. Grätzer, Lattice theory, Freeman, San Francisco, Calif., 1971.

4. _ Universal algebra, Van Nostrand, Princeton, N. J., 1968.

5. W. C. Holland, The lattice-ordered group of automorphisms of an ordred set, Michigan Math. J. 10 (1963), 399-408.

6. The largest proper variety of lattice-ordered groups, Proc. Amer. Math. Soc. 57 (1976), 25-28.

7. , Varieties of l-groups are torsion classess, Czechoslovak Math. J. 29 (104) (1979), 11-12.

8. J. T. Lloyd, Lattice-ordered groups and o-permutation groups, Dissertation, Tulane University, 1964.

9. J. Martinez, Free products in varieties of lattice-ordered groups, Czechoslovak Math. J. 22 (97) (1972), 535-553.

10. Varieties of lattice-ordered groups, Math. Z. 137 (1974), 265-284.

11. __ Torsion theory for lattice-ordered groups, Czechoslovak Math. J. 25 (100) (1975), 284-299. 
12. H. Neumann, Varieties of groups, Ergebnisse der Math. und ihrer Grenzgebiete, vol. 37, SpringerVerlag, New York, 1967.

13. E. B. Scrimger, A lange class of small varieties of lattice-ordered groups, Proc. Amer. Math. Soc. 51 (1975), 301-306.

14. E. C. Weinberg, Free lattice-ordered abelian groups. II, Math. Ann. 159 (1965), 217-222.

Departmient or Mathematics, Boise State University, Boise, IDaho 83725

Current address: Science and Mathematics Department, General Motors Institute, Flint, Michigan 48502 